\title{
Ring-like algebras associated with symmetricdifference
}

\author{
I. Chajda and H. Länger
}




\title{
RING-LIKE ALGEBRAS ASSOCIATED WITH SYMMETRIC DIFFERENCE
}

\author{
I. CHAJDA AND H. LÄNGER
}

Received 21 October, 2011

\begin{abstract}
We generalize the one-to-one correspondence between Boolean algebras and Boolean rings to so-called difference lattices and commutative strong difference ring-like algebras. Moreover, we show that difference ring-like algebras induce some sort of symmetric difference in corresponding posets.
\end{abstract}

2000 Mathematics Subject Classification: 06C15; 08A99

Keywords: poset, antitone involution, bounded lattice, generalized symmetric difference, difference ring-like algebra, induced poset, difference, difference lattice, strong difference ring-like algebra

\section{INTRODUCTION AND BASIC DEFINITIONS}

The well-known one-to-one correspondence between Boolean algebras and Boolean rings was generalized in many ways from Boolean algebras to more general structures (cf. e.g. [1],[2],[3],[4],[5] and [7],[9],[8],[10],[11]). This correspondence is realized via the so-called symmetric difference. A certain modification of this approach for orthocomplemented lattices is develope d e.g. in [11]. We already generalized the concept of symmetric difference for posets with an antitone involution in [6]. The aim of this paper is to generalize this correspondence to certain bounded lattices with an antitone involution. Moreover, we generalize Boolean rings in such a way that they induce some sort of symmetric difference on a certain bounded poset with an antitone involution.

We start our investigations with some basic definitions.

Definition 1. An antitone involution on a poset $(P, \leq)$ is a unary operation ' on $P$ satisfying

$$
x \leq y \text { implies } x^{\prime} \geq y^{\prime}
$$

Support of the research of the first and second author by ÖAD, Cooperation between Austria and Czech Republic in Science and Technology, grant No. CZ 01/2011, and of the first author by the Project CZ.1.07/2.3.00/20.0051 Algebraic Methods of Quantum Logicis is gratefully acknowledged. 


$$
\left(x^{\prime}\right)^{\prime}=x
$$

for all $x, y \in P$. A bounded poset with an antitone involution is an ordered quintuple $\left(P, \leq,{ }^{\prime}, 0,1\right)$ where $(P, \leq, 0,1)$ is a bounded poset and ' is an antitone involution on $(P, \leq)$. A bounded lattice with an antitone involution is an algebra $\left(L, \vee, \wedge,{ }^{\prime}, 0,1\right)$ of type $(2,2,1,0,0)$ such that $(L, \vee, \wedge, 0,1)$ is a bounded lattice and ' is an antitone involution on the poset $(L, \leq)$ corresponding to $(L, \vee, \wedge)$.

Remark 1. An algebra $\left(L, \vee, \wedge,{ }^{\prime}, 0,1\right)$ of type $(2,2,1,0,0)$ is a bounded lattice with an antitone involution if and only if $(L, \vee, \wedge, 0,1)$ is a bounded lattice and the de Morgan laws

$$
\begin{aligned}
& (x \vee y)^{\prime}=x^{\prime} \wedge y^{\prime} \\
& (x \wedge y)^{\prime}=x^{\prime} \vee y^{\prime}
\end{aligned}
$$

hold for all $x, y \in L$.

\section{DIFFERENCE RING-LIKE ALGEBRAS}

In this section we introduce certain generalizations of Boolean rings and show that they induce posets with an antitone involution where the addition plays the role of some sort of symmetric difference.

First we define a generalized symmetric difference on a bounded poset with an antitone involution.

Definition 2. A generalized symmetric difference on a bounded poset $\left(P, \leq,{ }^{\prime}, 0\right.$, 1) with an antitone involution is a binary operation + on $P$ satisfying the following axioms:

$$
\begin{aligned}
(x+y)+z & =x+(y+z) \\
x+y & =y+x \\
x+0 & =x \\
x+x & =0 \\
x+x^{\prime} & =1
\end{aligned}
$$

Remark 2. The operation + satisfies $x+1=x^{\prime}$ and $x+y=x^{\prime}+y^{\prime}$ since $x+1=$ $x+\left(x+x^{\prime}\right)=(x+x)+x^{\prime}=0+x^{\prime}=x^{\prime}+0=x^{\prime}$ and $x+y=(x+y)+0=$ $(x+y)+(1+1)=(x+1)+(y+1)=x^{\prime}+y^{\prime}$.

Now we define difference ring-like algebras.

Definition 3. A difference ring-like algebra is an algebra $(R,+, \cdot, 0,1)$ of type $(2,2,0,0)$ satisfying the following axioms:

$$
\begin{aligned}
(x+y)+z & =x+(y+z) \\
x+y & =y+x
\end{aligned}
$$




$$
\begin{aligned}
x+0 & =x \\
x+x & =0 \\
(x y) z & =x(y z) \\
x x & =x \\
x 1 & =x \\
1 x & =x \\
(x+1)(x y & +1)=x+1 \\
(x y+1)(x+1) & =x+1
\end{aligned}
$$

\begin{tabular}{|c|c|c|c|c|c|c|c|c|}
\hline+ & 0 & $a$ & $b$ & 1 & & 0 & $a$ & $b$ \\
\hline 0 & 0 & $a$ & $b$ & 1 & 0 & 0 & 0 & 0 \\
\hline$a$ & $a$ & 0 & 1 & $b$ & $a$ & 0 & $a$ & $a$ \\
\hline$b$ & $b$ & 1 & 0 & $a$ & $b$ & 0 & $b$ & $b$ \\
\hline 1 & 1 & $b$ & $a$ & 0 & 1 & 0 & $a$ & $b$ \\
\hline
\end{tabular}

$(R,+, \cdot, 0,1)$ is called a commutative difference ring-like algebra whenever the operation $\cdot$ is commutative.

Remark 3. There exist non-commutative difference ring-like algebras as the following example shows:

Example 1. If $R=\{0, a, b, 1\}$ and + and $\cdot$ are defined by

then $(R,+, \cdot, 0,1)$ is a non-commutative difference ring-like algebra since $a b=a \neq$ $b=b a$.

Now we can prove that every difference ring-like algebra induces in a natural way a generalized symmetric difference on a certain bounded poset with an antitone involution.

Theorem 1. If $(R,+, \cdot, 0,1)$ is a difference ring-like algebra then there exists a bounded poset $\mathcal{P}=\left(R, \leq,{ }^{\prime}, 0,1\right)$ with an antitone involution such that $+i$ is a generalized symmetric difference on $\mathcal{P}$.

Proof. Define $x \leq y$ if $x y=y x=x(x, y \in R)$ and $x^{\prime}:=x+1$ for all $x \in R$. Then $(R, \leq)$ is a poset with greatest element 1 . Now let $a, b, c \in R$. If $a \leq b$ then $a b=b a=a,(b+1)(a+1)=(b+1)(b a+1)=b+1$ according to (2.1) and $(a+1)(b+1)=(b a+1)(b+1)=b+1$ according to (2.2), i. e. $b+1 \leq a+1$. Moreover, $(a+1)+1=a+(1+1)=a+0=a$. Hence ${ }^{\prime}$ is an antitone involution on $(R, \leq)$ and $1^{\prime}=1+1=0$ its least element. We have proved that $\mathcal{P}:=\left(R, \leq,^{\prime}, 0,1\right)$ is a bounded poset with an antitone involution. Finally, we have

$$
\begin{aligned}
(a+b)+c & =a+(b+c), \\
a+b & =b+a,
\end{aligned}
$$




$$
\begin{aligned}
& a+0=a \\
& a+a=0 \text { and } \\
& a+1=a^{\prime} \text { by definition. }
\end{aligned}
$$

From here we infer

$$
a+a^{\prime}=a+(a+1)=(a+a)+1=0+1=1+0=1 .
$$

Definition 4. The bounded poset $(R, \leq, 0,1)$ constructed in the proof of Theorem 1 will be called the poset induced by the difference ring-like algebra $(R,+, \cdot, 0,1)$.

\begin{tabular}{|c|c|c|c|c|c|c|c|c|}
\hline+ & 0 & $a$ & $b$ & 1 & . & 0 & $a$ & $b$ \\
\hline 0 & 0 & $a$ & $b$ & 1 & 0 & 0 & 0 & 0 \\
\hline$a$ & $a$ & 0 & 1 & $b$ & $a$ & 0 & $a$ & $a$ \\
\hline$b$ & $b$ & 1 & 0 & $a$ & $b$ & 0 & $a$ & $b$ \\
\hline 1 & 1 & $b$ & $a$ & 0 & 1 & 0 & $a$ & $b$ \\
\hline
\end{tabular}

Remark 4. Every Boolean ring is a commutative difference ring-like algebra. That the converse is not true can be seen from the following example:

Example 2. If $R=\{0, a, b, 1\}$ and + and $\cdot$ are defined by

then $(R,+, \cdot, 0,1)$ is a commutative difference ring-like algebra that is not a Boolean ring since

$$
(a+b) b=1 \cdot b=b \neq 1=a+b=a b+b b .
$$

Moreover, the induced poset is the chain $0<a<b<1$ and the involution' is the permutation $(01)(a b)$.

Next we show that every commutative difference ring-like algebra already induces a generalized symmetric difference on a bounded lattice with an antitone involution.

Theorem 2. If $(R,+, \cdot, 0,1)$ is a commutative difference ring-like algebra and

$$
\begin{aligned}
x \vee y & :=(x+1)(y+1)+1 \\
x \wedge y & :=x y \text { and } \\
x^{\prime} & :=x+1
\end{aligned}
$$

for all $x, y \in R$ then $\mathscr{L}:=\left(R, \vee, \wedge,{ }^{\prime}, 0,1\right)$ is a bounded lattice with an antitone involution and + is a generalized symmetric differenc e on $\mathscr{L}$.

Proof. The proof follows the same lines as that of Theorem 1. However, $x \leq y$ is now equivalent to $x y=x(x, y \in R)$ and $(R, \cdot)$ is a meet-semilattic e the corresponding poset of which is $(R, \leq)$. Since $\left(R, \leq,{ }^{\prime}, 0,1\right)$ is a bounded poset with an antitone involution and $(R, \wedge)$ with $x \wedge y:=x y$ for all $x, y \in R$ the corresponding meet-semilattice, $\left(R, \vee, \wedge,{ }^{\prime}, 0,1\right)$ with $x \vee y ;=(x+1)(y+1)+1$ for all $x, y \in R$ is a bounded lattice with an antitone involution. The rest follows from the proof of Theorem 1. 


\section{DifFERENCE LATTICES AND COMMUTATIVE STRONG DIFFERENCE RING-LIKE ALGEBRAS}

In this section we want to derive a one-to-one correspondence between certain lattices with some kind of symmetric difference and certain ring-like algebras generalizing the well-known one-to-one correspondence between Boolean algebras and Boolean rings. We generalize the approach by Matoušek and Pták (cf. [11]).

First we define the lattice structures.

Definition 5. A difference on a bounded lattice $\left(L, \vee, \wedge,{ }^{\prime}, 0,1\right)$ with an antitone involution is a binary operation $\triangle$ on $L$ satisfying the following axioms:

$$
\begin{aligned}
(x \triangle y) \triangle z & =x \triangle(y \triangle z) \\
x \triangle 1 & =x^{\prime} \\
1 \triangle x & =x^{\prime} \\
x \triangle x & =0 \\
x \triangle y & \leq x \vee y
\end{aligned}
$$

A difference lattice is an algebra $\left(L, \vee, \wedge, \triangle,^{\prime}, 0,1\right)$ of type $(2,2,1,0,0,2)$ such that $\mathscr{L}=\left(L, \vee, \wedge,{ }^{\prime}, 0,1\right)$ is a bounded lattice with an antitone involution and $\triangle$ is a difference on $\mathscr{L}$.

Remark 5. If $\left(B, \vee, \wedge,{ }^{\prime}, 0,1\right)$ is a Boolean algebra and $\triangle$ denotes the usual symmetric difference given by $x \triangle y=\left(x^{\prime} \wedge y\right) \vee\left(x \wedge y^{\prime}\right)=(x \vee y) \wedge\left(x^{\prime} \vee y^{\prime}\right)$ then $\left(B, \vee, \wedge, \triangle,^{\prime}, 0,1\right)$ is a differenc e lattice with an antitone involution. That the converse is not true is shown by the following example:

Example 3. If $L=\{0, a, b, c, d, e, f, 1\}$ and $\vee, \wedge,{ }^{\prime}$ and $\triangle$ are defined by the tables

\begin{tabular}{l|llllllll}
$\vee$ & 0 & $a$ & $b$ & $c$ & $d$ & $e$ & $f$ & 1 \\
\hline 0 & 0 & $a$ & $b$ & $c$ & $d$ & $e$ & $f$ & 1 \\
$a$ & $a$ & $a$ & 1 & 1 & 1 & 1 & 1 & 1 \\
$b$ & $b$ & 1 & $b$ & 1 & 1 & 1 & 1 & 1 \\
$c$ & $c$ & 1 & 1 & $c$ & 1 & 1 & 1 & 1 \\
$d$ & $d$ & 1 & 1 & 1 & $d$ & 1 & 1 & 1 \\
$e$ & $e$ & 1 & 1 & 1 & 1 & $e$ & 1 & 1 \\
$f$ & $f$ & 1 & 1 & 1 & 1 & 1 & $f$ & 1 \\
1 & 1 & 1 & 1 & 1 & 1 & 1 & 1 & 1
\end{tabular}

\begin{tabular}{l|llllllll|l}
$\wedge$ & 0 & $a$ & $b$ & $c$ & $d$ & $e$ & $f$ & 1 & \\
\hline 0 & 0 & 0 & 0 & 0 & 0 & 0 & 0 & 0 & 1 \\
$a$ & 0 & $a$ & 0 & 0 & 0 & 0 & 0 & $a$ & $f$ \\
$b$ & 0 & 0 & $b$ & 0 & 0 & 0 & 0 & $b$ & $e$ \\
$c$ & 0 & 0 & 0 & $c$ & 0 & 0 & 0 & $c$ & $d$ \\
$d$ & 0 & 0 & 0 & 0 & $d$ & 0 & 0 & $d$ & $c$ \\
$e$ & 0 & 0 & 0 & 0 & 0 & $e$ & 0 & $e$ & $b$ \\
$f$ & 0 & 0 & 0 & 0 & 0 & 0 & $f$ & $f$ & $a$ \\
1 & 0 & $a$ & $b$ & $c$ & $d$ & $e$ & $f$ & 1 & 0
\end{tabular}




\begin{tabular}{l|llllllll}
$\triangle$ & 0 & $a$ & $b$ & $c$ & $d$ & $e$ & $f$ & 1 \\
\hline 0 & 0 & $a$ & $b$ & $c$ & $d$ & $e$ & $f$ & 1 \\
$a$ & $a$ & 0 & $c$ & $b$ & $e$ & $d$ & 1 & $f$ \\
$b$ & $b$ & $c$ & 0 & $a$ & $f$ & 1 & $d$ & $e$ \\
$c$ & $c$ & $b$ & $a$ & 0 & 1 & $f$ & $e$ & $d$ \\
$d$ & $d$ & $e$ & $f$ & 1 & 0 & $a$ & $b$ & $c$ \\
$e$ & $e$ & $d$ & 1 & $f$ & $a$ & 0 & $c$ & $b$ \\
$f$ & $f$ & 1 & $d$ & $e$ & $b$ & $c$ & 0 & $a$ \\
1 & 1 & $f$ & $e$ & $d$ & $c$ & $b$ & $a$ & 0
\end{tabular}

then $\left(L, \vee, \wedge, \triangle,^{\prime}, 0,1\right)$ is a difference lattice such that $\left(L\right.$, vee, $\left.,,^{\prime}, 0,1\right)$ is not a Boolean algebra since

$$
(a \vee b) \wedge c=1 \wedge c=c \neq 0=0 \vee 0=(a \wedge c) \vee(b \wedge c) .
$$

Next we define the ring-like counterpart.

Definition 6. A difference ring-like algebra $(R,+, \cdot, 0,1)$ is called strong if it satisfies the axiom

$$
(x+y)(x y+1)=x+y .
$$

Remark 6. Every Boolean ring is a commutative strong difference ring-like algebra. That the converse is not true is shown by the following example:

Example 4. Let $R=\{0, a, b, c, d, e, f, 1\}$ and define + and $\cdot$ as $\triangle$ and $\wedge$ in Example 3 , respectively. Then $(R,+, \cdot, 0,1)$ is a commutati ve strong difference ringlike algebra that is not a Boolean ring since $(a+f) b=1 \cdot b=b \neq 0=0+0=$ $a b+f b$.

Now we can prove that every difference lattice induces a commutative strong difference ring-like algebra in a natural way.

Theorem 3. If $\mathscr{L}=\left(L, \vee, \wedge, \triangle,^{\prime}, 0,1\right)$ is a difference lattice and one defines

$$
\begin{aligned}
x+y & :=x \triangle y \text { and } \\
x y & :=x \wedge y
\end{aligned}
$$

for all $x, y \in L$ then $\mathbf{R}(\mathscr{L}):=(L,+, \cdot, 0,1)$ is a commutative strong difference ringlike algebra.

Proof. Let $\mathscr{L}=\left(L, \vee, \wedge, \triangle,^{\prime}, 0,1\right)$ be a difference lattice, define

$$
\begin{aligned}
x+y & :=x \Delta y \text { and } \\
x y & :=x \wedge y
\end{aligned}
$$

for all $x, y \in L$ and let $a, b, c \in L$. Then

$$
(a+b)+c=(a \triangle b) \triangle c=a \triangle(b \triangle c)=a+(b+c),
$$




$$
\begin{aligned}
a+1 & =a \triangle 1=a^{\prime}, \\
1+a & =1 \triangle a=a^{\prime}, \\
a+a & =a \triangle a=0, \\
a+0 & =a+(1+1)=(a+1)+1=\left(a^{\prime}\right)^{\prime}=a, \\
0+a & =(1+1)+a=1+(1+a)=\left(a^{\prime}\right)^{\prime}=a, \\
a+b & =(a+b)+0=(a+b)+((b+a)+(b+a))= \\
& =((a+(b+b))+a)+(b+a)=((a+0)+a)+(b+a)= \\
& =(a+a)+(b+a)=0+(b+a)=b+a, \\
(a b) c & =(a \wedge b) \wedge c=a \wedge(b \wedge c)=a(b c), \\
a b & =a \wedge b=b \wedge a=b a, \\
a a & =a \wedge a=a, \\
a 1 & =a \wedge 1=a, \\
(a+1)(a b+1) & =a^{\prime} \wedge(a \wedge b)^{\prime}=a^{\prime}=a+1, \\
a^{\prime}+b^{\prime} & =(a+1)+(1+b)=(a+(1+1))+b=(a+0)+b= \\
& =a+b \text { and } \\
(a+b)(a b+1) & =(a+b) \wedge(a \wedge b)^{\prime}=\left(a^{\prime}+b^{\prime}\right) \wedge\left(a^{\prime} \vee b^{\prime}\right)= \\
& =\left(a^{\prime} \triangle b^{\prime}\right) \wedge\left(a^{\prime} \vee b^{\prime}\right)=a^{\prime} \triangle b^{\prime}=a^{\prime}+b^{\prime}=a+b \text { according to }
\end{aligned}
$$

Hence $\mathbf{R}(\mathscr{L})=(L,+, \cdot, 0,1)$ is a commutative strong difference ring-like algebra.

We can also prove the converse, namely that every commutative strong difference ring-like algebra induces in a natural way a difference lattice.

Theorem 4. If $\mathcal{R}=(R,+, \cdot, 0,1)$ is a commutative strong difference ring-like algebra and

$$
\begin{aligned}
x \vee y & :=(x+1)(y+1)+1, \\
x \wedge y & :=x y, \\
x^{\prime} & :=x+1 \text { and } \\
x \triangle y & :=x+y
\end{aligned}
$$

for all $x, y \in R$ then $\mathbf{L}(\mathcal{R}):=\left(R, \vee, \wedge, \triangle,{ }^{\prime}, 0,1\right)$ is a difference lattice.

Proof. Let $\mathcal{R}=(R,+, \cdot, 0,1)$ be a commutative strong difference ring-like algebra, define

$$
x \vee y:=(x+1)(y+1)+1,
$$




$$
\begin{aligned}
x \wedge y & :=x y, \\
x^{\prime} & :=x+1 \text { and } \\
x \triangle y & :=x+y
\end{aligned}
$$

for all $x, y \in R$ and let $a, b, c \in R$. According to Theorem $2,\left(R, \vee, \wedge,{ }^{\prime}, 0,1\right)$ is a bounded lattice with an antitone involution. Moreover, we have

$$
\begin{aligned}
(a \triangle b) \triangle c & =(a+b)+c=a+(b+c)=a \triangle(b \triangle c), \\
a \triangle 1 & =a+1=a^{\prime}, \\
1 \triangle a & =1+a=a+1=a^{\prime}, \\
a \triangle a & =a+a=0 \text { and } \\
a \triangle b & =a+b=(a+b)+0=(a+b)+(1+1)=(a+1)+(b+1)= \\
& =((a+1)+(b+1))((a+1)(b+1)+1)= \\
& =((a+1)+(b+1)) \wedge(a \vee b) \leq a \vee b .
\end{aligned}
$$

Hence $\mathbf{L}(\mathcal{R})=\left(R, \vee, \wedge, \triangle,^{\prime}, 0,1\right)$ is a difference lattice.

We can now show that the correspondence between difference lattices and commutative strong difference ring-like algebras described in Theorems 3 and 4 is oneto-one.

\section{Theorem 5.}

(i) $\mathbf{L}(\mathbf{R}(\mathscr{L}))=\mathscr{L}$ for every differen ce lattice $\mathscr{L}$.

(ii) $\mathbf{R}(\mathbf{L}(\mathcal{R}))=\mathcal{R}$ for every commutative strong difference ring-like algebra $\mathcal{R}$.

Proof.

(i) If $\mathscr{L}=\left(L, \vee, \wedge, \triangle,^{\prime}, 0,1\right)$ is a difference lattice, $\mathbf{R}(\mathscr{L})=(L,+, \cdot, 0,1), \mathbf{L}(\mathbf{R}(\mathscr{L}))=$ $\left(L, \cup, \cap, \circ,{ }^{*}, 0,1\right)$ and $a, b \in L$ then

$$
\begin{aligned}
a \cup b & =(a+1)(b+1)+1=((a \triangle 1) \wedge(b \triangle 1)) \triangle 1=\left(a^{\prime} \wedge b^{\prime}\right)^{\prime}=a \vee b, \\
a \cap b & =a b=a \wedge b, \\
a \circ b & =a+b=a \triangle b \text { and } \\
a^{*} & =a+1=a \triangle 1=a^{\prime} .
\end{aligned}
$$

(ii) If $\mathcal{R}=(R,+, \cdot, 0,1)$ is a commutative strong difference ring-like algebra, $\mathbf{L}(\mathcal{R})=\left(R, \vee, \wedge, \triangle,^{\prime}, 0,1\right)$, bf $R(\mathbf{L}(\mathcal{R}))=(R, \oplus, \odot, 0,1)$ and $a, b \in R$ then

$$
\begin{aligned}
& a \oplus b=a \triangle b=a+b \text { and } \\
& a \odot b=a \wedge b=a b .
\end{aligned}
$$




\section{FURTHER EXAMPLES}

In this section we generalize the examples given before.

Example 5. If $n \geq 2, R:=\{0,1\}^{n}, 0:=(0, \ldots, 0), 1:=(1, \ldots, 1),+$ the componentwise addition modulo $2,0 x=x 0:=0$ and $x 1=1 x:=x$ for all $x \in R$ and $x y:=x$ for all $x, y \in R \backslash\{0,1\}$ then $(R,+, \cdot, 0,1)$ is a non- commutative difference ring-like algebra since

$$
(0, \ldots, 0,1)(1, \ldots, 1,0)=(0, \ldots, 0,1) \neq(1, \ldots, 1,0)=(1, \ldots, 1,0)(0, \ldots, 0,1) .
$$

For $n=2$ we obtain Example 1.

Example 6. If $n \geq 2, R:=\{0,1\}^{n}, 0:=(0, \ldots, 0), 1:=(1, \ldots, 1),+$ the componentwise addition modulo 2 and - the minimum with respect to the lexicographical order then $(R,+, \cdot, 0,1)$ is a commutative difference ring- like algebra that is not a Boolean ring since

$$
\begin{aligned}
& ((0, \ldots, 0,1)+(1, \ldots, 1,0))(1, \ldots, 1,0)= \\
& =1 \cdot(1, \ldots, 1,0)=(1, \ldots, 1,0) \neq 1=(0, \ldots, 0,1)+(1, \ldots, 1,0)= \\
& =(0, \ldots, 0,1)(1, \ldots, 1,0)+(1, \ldots, 1,0)(1, \ldots, 1,0) .
\end{aligned}
$$

For $n=2$ we obtain Example 2.

Example 7. If $n \geq 3, L:=\{0,1\}^{n}, 0:=(0, \ldots, 0), 1:=(1, \ldots, 1), x \vee x=x$ vee $0=$ $0 \vee x:=x$ for all $x \in L, x \vee y:=1$ for all $x, y \in L \backslash\{0\}$ with $x \neq y, x \wedge x=$ $x \wedge 1=1 \wedge x:=x$ for all $x \in L, x \wedge y:=0$ for all $x, y \in L \backslash\{1\}$ with $x \neq y, \triangle$ denotes the componentwise addition modulo 2 and $x^{\prime}:=x \triangle 1$ for all $x \in L$ then $\left(L, \vee, \wedge, \triangle,^{\prime}, 0,1\right)$ is a difference lattice such that $\left(L, \vee, \wedge,{ }^{\prime}, 0,1\right)$ is not a Boolean algebra since

$$
\begin{aligned}
& ((0, \ldots, 0,1) \vee(0, \ldots, 0,1,0)) \wedge(0, \ldots, 0,1,1)= \\
& =1 \wedge(0, \ldots, 0,1,1)=(0, \ldots, 0,1,1) \neq 0=0 \vee 0= \\
& =((0, \ldots, 0,1) \wedge(0, \ldots, 0,1,1)) \vee((0, \ldots, 0,1,0) \wedge(0, \ldots, 0,1,1)) .
\end{aligned}
$$

For $n=3$ we obtain Example 3 .

Example 8. If $n \geq 3, R:=\{0,1\}^{n}, 0:=(0, \ldots, 0), 1:=(1, \ldots, 1)$ and + and $\cdot$ are defined as $\triangle$ and $\wedge$ in Example 7 , respectively, then $(R,+, \cdot, 0,1)$ is a commutative strong difference ring-like algebra that is not a Boolean ring since

$$
\begin{aligned}
& ((0, \ldots, 0,1)+(1, \ldots, 1,0))(0, \ldots, 0,1,0)= \\
& =1 \cdot(0, \ldots, 0,1,0)=(0, \ldots, 0,1,0) \neq 0=0+0= \\
& =(0, \ldots, 0,1)(0, \ldots, 0,1,0)+(1, \ldots, 1,0)(0, \ldots, 0,1,0) .
\end{aligned}
$$

This commutative strong difference ring-like algebra corresponds to the difference lattice defined in Example 7. For $n=3$ we obtain Example 4. 


\section{REFERENCES}

[1] I. Chajda and G. Eigenthaler, "A note on orthopseudorings and Boolean quasirings," Sitzungsber., Abt. II, Österr. Akad. Wiss., Math.-Naturwiss. Kl., vol. 207, pp. 83-94, 1998.

[2] I. Chajda and H. Länger, "Orthorings," Discuss. Math., Gen. Algebra Appl., vol. 24, no. 1, pp. 137-147, 2004.

[3] I. Chajda and H. Länger, "Ring-like structures corresponding to MV-algebras via symmetric difference," Sitzungsber., Abt. II, Österr. Akad. Wiss., Math.-Naturwiss. Kl., vol. 213, pp. 33-41, 2004.

[4] I. Chajda and H. Länger, "Hereditary generalized Boolean quasirings, MV-algebras and De Morgan algebras," in Proceedings of the 70th workshop on general algebra "70. Arbeitstagung Allgemeine Algebra", G. Dorfer, Ed. Klagenfurt: Verlag Johannes Heyn, 2006, pp. 31-40.

[5] I. Chajda and H. Länger, "A common generalization of ortholattices and Boolean quasirings," Demonstr. Math., vol. 40, no. 4, pp. 769-774, 2007.

[6] I. Chajda and H. Länger, "Symmetric differences on posets with an antitone involution," Order, vol. 29, no. 1, pp. 215-225, 2012.

[7] I. Chajda, H. Länger, and M. Msaczyński, "Ring-like structures corresponding to generalized orthomodular lattices," Math. Slovaca, vol. 54, no. 2, pp. 143-150, 2004.

[8] D. Dorninger, H. Länger, and M. Maczyński, "On ring-like structures occurring in axiomatic quantum mechanics," Sitzungsber., Abt. II, Österr. Akad. Wiss., Math.-Naturwiss. Kl., vol. 206, pp. 279-289, 1997.

[9] D. Dorninger, H. Länger, and M. Mączyński, "The logic induced by a system of homomorphisms and its various algebraic characterizations," Demonstr. Math., vol. 30, no. 1, pp. 215-232, 1997.

[10] H. Länger, "Generalizations of the correspondence between Boolean algebras and Boolean rings to orthomodular lattices," Tatra Mt. Math. Publ., vol. 15, pp. 97-105, 1998.

[11] M. Matoušek and P. Pták, "Orthocomplemented difference lattices with few generators," Kybernetika, vol. 47, no. 1, pp. 60-73, 2011.

\section{Authors' addresses}

\section{Chajda}

Palacký University Olomouc, Department of Algebra and Geometry, 17. Listopadu 12, 77146 Olomouc, Czech Republic

E-mail address: ivan.chajda@upol.cz

\section{H. Länger}

Vienna University of Technology, Institute of Discrete Mathematics and Geometry, Wiedner Hauptstraße 8-10, 1040 Vienna, Austria

E-mail address: h. laengeretuwien.ac.at 